\title{
The Research of Internet-Based Health Management for the Elderly
}

\author{
Kaijun Zhang*, Weidong He, Jiayu Zhou \\ College of Marketing, Nanjing University of Finances and Economics, Nanjing, China \\ Email address \\ 519425207@qq.com (Kaijun Zhang) \\ ${ }^{*}$ Corresponding author
}

\section{To cite this article:}

Kaijun Zhang, Weidong He, Jiayu Zhou. The Research of Internet-Based Health Management for the Elderly. Science Discovery. Vol. 4, No. 4, 2016, pp. 220-225. doi: 10.11648/j.sd.20160404.13

Received: June 12, 2016; Accepted: July 6, 2016; Published: July 20, 2016

\begin{abstract}
At present, China has fully entered the aging society, which brings severe challenges to the development of China's economic and the management of policy. As the health management of elderly relate to every family, a questionnaire survey was carried out from the health issues of elderly which was concerned by the society. The survey conclude the number of homes for the elderly, the number of senior citizens who suffering from chronic diseases, the type of diseases, people's understanding of the disease and diet control, food service demand and so on. The questionnaires were sent by internet, and finally got the data for statistical analysis. The results show that most families have 1-4 elderly, chronic age-related diseases such as hyperglycemia, hypertension, hyperlipemia, heart disease and other diseases which are confusing and affecting the lives of every family. For this reason the elderly group shows a great demand for healthy catering. The essay which combines with the survey results and current research of the Internet will analyze and discuss a feasible approach to solve the problem in elderly health management. While the solution of connecting Internet with health management meets the needs of society, it also has a great developing potential, and even high feasibility and operability when it comes into operation.
\end{abstract}

Keywords: Current Situation of Aging, Health Management System, Diet, Development of Internet, Wearable Devices

\section{基于互联网的老年人健康管理研究}

张凯俊 ${ }^{*}$ 何卫东, 周珈羽

市场营销与物流管理学院, 南京财经大学, 南京, 中国

邮箱

519425207@qq. com（张凯俊）

摘要: 目前中国已全面步入老龄化社会, 老龄化将带给中国经济和政策层面的严峻挑战, 老年人的健康管理关系着每一 个家庭。本论文从社会关注的老年人健康问题展开问卷调查, 问卷内容包括家庭老人人数, 患慢性疾病人数, 类型, 疾病知晓, 饮食控制了解度, 饮食服务需求等内容。问卷采用网络问卷形式, 最后获取数据进行统计分析。结果显示, 大部分家庭拥有 1 到 4 个老人, 慢性老年疾病如高血糖, 高血压, 高血脂, 心脏病等正困惑及影响每个家庭的生活, 老 年人群体对于健康配餐有着极大需求。本论文通过问卷调查结果, 结合目前互联网发展势头, 分析探讨一条解决老年 健康问题管理的可行性办法, 认为互联网+健康管理符合社会需求, 有很大发展潜力及发展空间, 具体实施起来也具 有较高的可行性和可操作性。

关键词：老龄化现状, 健康管理系统, 饮食搭配, 互联网发展, 可穿戴设备 


\section{1. 引言}

本论文从社会关注的老年人健康问题展开问卷调查, 旨在探索一条解决老年健康问题的可行性办法。

\section{1. 老龄化现状}

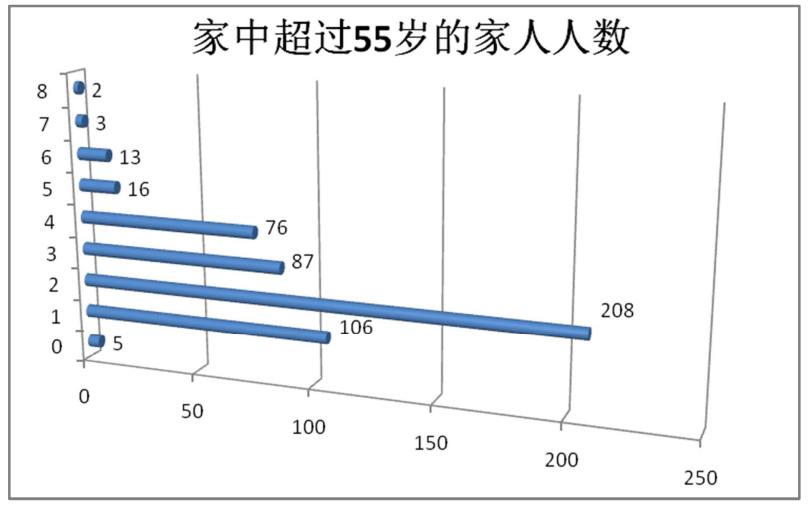

图1 普通家庭老年人数量图。

目前我国 60 岁以上老年人口已达 1.44 亿，占总人口 的 $11 \%$ 。中国已全面步入老龄化社会 [1], 老龄化将带给中 国经济和政策层面的严峻挑战, 这不仅包括给社会医疗保 障体制、经济、基础设施建设、社会精神文明建设带来压 力, 还会使政府将更多的钱投到 “乘数效应” 低的社会福 利, 相对降低了效率高的发展领域的投资, 导致整体经济 的发展速度成倍地减慢。此外, 人口老龄化还会带来老年 人家庭代际结构的变化以及对传统的养老模式的冲击。中 国的老龄化趋势所带来的问题不容小䖒。

根据问卷调查结果，可以看到: 大部分家庭家中都有 超过 55 岁的家人，其人数为 $1-4$ 个，而家中没有老年人的 比例仅

仅为 $5 \%$ 不到, 这也意味着在当代中国社会, 至少有 $95 \%$ 以上的家庭承担着赡养老年人的负担。双亲是老年人的家 庭数量占样本数量的 $40 \%$, 老年人的数量在逐步上升。通 过分析图形走势可以看出, 未来大部分中国家庭家中老年 人的数量将在 3 到 4 个, 养老负担将大大增加。

\section{2. 老年人的健康现状}

2005年世界卫生组织发表了题为《预防慢性病: 一项 至关重要的投资》的全球性报告, 报告指出, 目前慢性病 是世界上最首要的死亡原因, 由慢性病造成的死亡约占所 有死亡的 $60 \%$ [2]。慢性病, 全名为慢性非传染性疾病, 指发病隐匿、长期的、不能自愈的、很难治愈的一组疾病, 包括高血压、糖尿病、高脂血症、慢性阻塞性肺部疾病、 恶性肿瘤等慢性病 [3]。具有高发病率、高患病率、高致 残率、高死亡率和医疗费用昂贵的特点, 它严重影响居民 的生存质量。而老年人群随着机体功能的逐渐衰退, 是患 慢性病的主要人群, 慢性病严重威胁老年人健康, 已成为 老年人的主要公共卫生问题。

在516份调查问卷中, 有超过半数的调查对象反映家 里的老年人正面临着严重的三高问题。

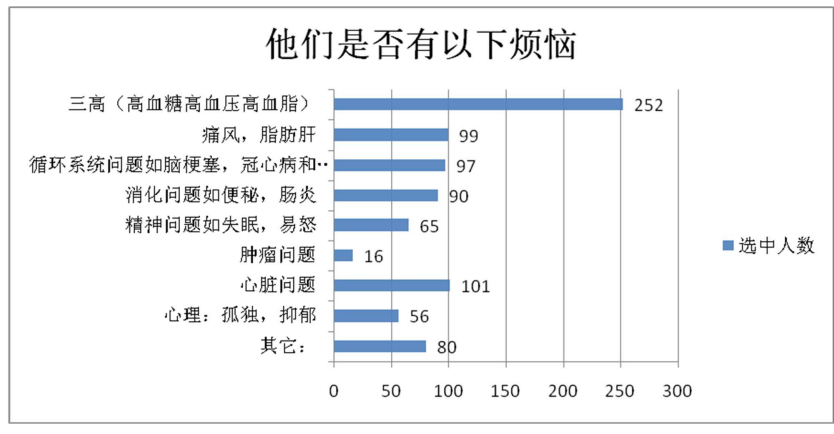

图2 老年人疾病及患病人数图。

高血压, 高血脂, 高血糖是中国老年人最常患的代谢 综合征, 而跟三高息息相关的糖尿病, 动脉硬化, 肥胖, 心脑血管疾病是人类致命的 “头号杀手”。在中国人的十 大死亡原因中, 与代谢疾病相关的死亡率就高达 $35.7 \%$, 与 “三高” 相关的死亡人数就占总死亡人数的 $27 \%$ [4]

在所有调查对象中, 有 $20 \%$ 的老年人承担着心脏问题 的危险, 有 $19 \%$ 的老年人受着痛风, 脂肪肝的影响, $18 \%$ 的老年人受循环系统疾病的困扰, 还有 $17 \%$ 的老年人饱受 消化疾病的痛, 除此之外, 心理问题, 精神问题, 肿瘤 问题都时刻威胁着老年人的健康。更为令人担忧的是, 老年人所患的疾病并不是单一存在的, 许多老年人是高 血压高血糖高血脂, 循环系统问题, 心脏问题并存, 这 大大增加了健康风险和治疗难度。老年人的健康现状十 分严峻。

\section{3. 互联网现状}

截至2015年6月，我国网民规模达6.68亿，互联网普 及率为 $48.8 \%$, 较 2014 年底提升了 0.9 个百分点, 整体网民 规模增速继续放缓 [5]。然而从现状来看, “互联网+”处 于初级阶段——在热谈但没有落实的理论阶段, 特别是在 老年人健康方面, 互联网与老年人健康的结合非常差, 打 开APP应用市场和调查现有的操作软件系统, 可以看到针 对老年人的软件硬件非常少, 绝大部分停滞于老年人手机、 老年人输入法、老年人桌面等, 没有真正为老年人健康所 着想的软件硬件系统。而国内的移动互联网医疗应用总体 处于起步阶段, 2013年8月, 通过手机搜索 “医疗” 关键 词可以搜到 1500 个手机客户端应用程序, 搜索 “健康” 达 5400 个。这些应用涉及的范围非常广泛，有医疗咨询、诊 疗知识宝典、预约挂号、健康自测、药物和疾病手册、急 救助手、手机药店、慢病管理等等。

目前, 国内相对有一定知名度的应用有三种: 首先 是医患交互型, 这种类型的应用最出名的是 “春雨掌上 医生”, 它是一款专业手机医生问答软件, 通过建立疾 病数据库和整合医生资源, 为用户提供移动的自诊或在 线问诊服务; 其次是知识辅助型。如 “全科医生” 这一 类软件, 是面向专业医护人员开发的掌上信息查询及决 策工具平台; 最后还有综合服务型。如 “移动医院” 则 是一款利用智能手机终端由医院为患者提供的综合医疗 服务平台。 


\section{2. 健康管理系统}

\section{1. 研究方法}

通过制定与老年人健康相关的问卷，包括家庭老人人 数, 患慢性疾病人数, 类型, 疾病知晓, 饮食控制了解度, 饮食服务需求等内容, 采用网络问卷形式, 最后获取数据 进行统计分析, 根据分析结果判断健康管理系统可行性。

\section{2. 理论提出}

\subsection{1. 「互联网 + 」与健康管理的有效结合}

随着中国步入老龄化社会, 一系列健康问题开始困扰 着老年人, 其中最突出的便是慢性病。然而慢病管理是一 个庞大的系统工程, 相关政策早就提出, 应通过以慢性病 防治为切入点, 建立分级诊疗制度, 引导群众科学有序就 医, 强化对慢性病诊治的规范管理。一方面, 与医疗资源 集中、患者人潮汹涌的大医院相比, 基层医疗机构显然更 适合承担慢病管理的职责。基层医疗机构的医护人员能够 拥有足够的时间与患者进行充分的交流，对患者的身体状 况和病史获得全面的了解, 进而建立长期持续的联系, 将 医患双方变成协同合作的平等伙伴。另一方面, 慢性病管 理更多的是改变人民群众的的行为习惯、饮食、运动、监 测, 同时要加强自我管理的能力。在这方面, 互联网及移 动互联网技术大有可为, 例如通过「互联网+」构建个人 健康管理档案, 实现「可延续」「可转移」的立体数据库, 帮助患者建立全方位的健康数据体征。同时，通过「互联 网+」智能硬件, 方便对患者的各项健康数据进行查阅、 分析, 大大简化基层慢病管理工作，提高基层数据化决策 水平。多项实践模式也表明, 只有解决基层缺医少药问题, 慢病管理才能真正下沉到基层。而这些难题都可以通过 $\lceil$ 互联网 $+」$ 手段不断缓解、优化, 并得到有效应对。[6]

\section{2. 2. 基于互联网建立健康管理系统}

i. 家庭担忧

在中国, 与老年人健康相关的医疗监控体系和医疗保 障仍未完全建立, 医疗体系存在着许多问题, 如医疗服务 的公平性下降，卫生投入的宏观效率低下，医疗服务体系 不适应群众的健康需求, 医疗保险的覆盖率过低、强制性 不够、预防功能不强等。

根据调查结果可以看出:

\section{您是否担心家里老人每天的不当饮食会给} 老人身体带来危害?
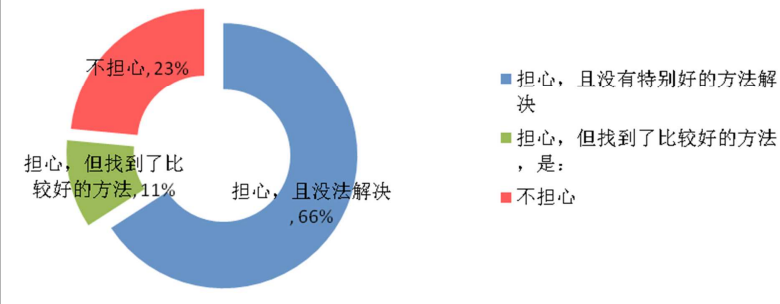

图3 老年人饮食担忧比例图。
在516个受访对象中，有341个家庭（66\%）由于缺乏 基本的医疗饮食保障和知识, 正担心着老年人的日常饮食 不当会对老年人身体造成危害。由于中国的健康医疗系统 不完善, 基础健康营养教育贵乏, 同时在逐步建立起医疗 保障系统的过程中, 忽略了对日常饮食的控制和日常健康 的教育, 老年人经常面临饮食健康问题。而从长远角度来 说, 日常控制和教育是最能帮助老年人管理健康的工具。

ii. 选择的疑惑

大部分中老年人对于食物的认知仅存在于上火与不 上火, 油淢与不油淢, 高糖与低糖这一些模糊的概念, 对 于如何搭配日常饮食, 如何做到三大项: 碳水化合物, 蛋 白质, 脂肪的合理搭配以及微量元素的补充都不甚清楚。 随着中国经济的不断发展, 对于大部分家庭来说, 日常饮 食不再是有什么吃什么, 而是什么应该吃、什么不能吃、 以及什么少吃点。但许多人无法实现这简单的三步, 所以 往往这些不合理的膡食结构就会导致疾病的发生。

从问卷结果可以看出, 有 $21 \%$ 的家庭无法正常进行日 常饮食搭配, 而有 $73 \%$ 的家庭虽然知道不能吃什么, 但在 面临市面上纷杂的食品保健品时也是难以抉择。

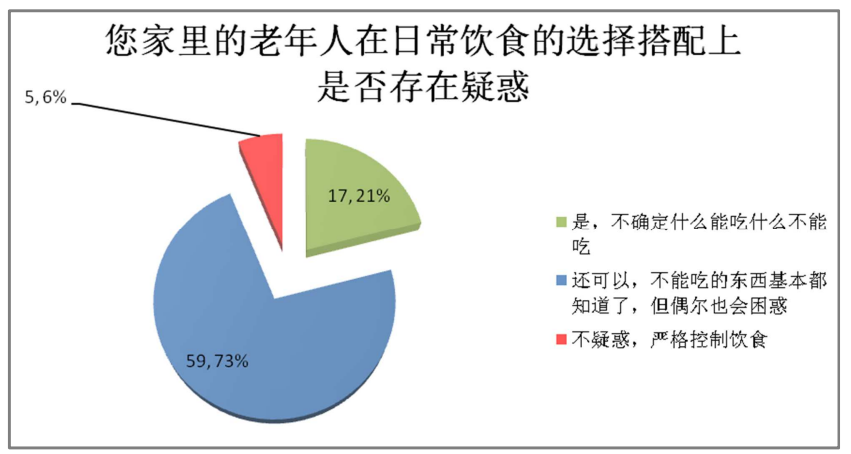

图4 老年人饮食困惑比例图。

只有极少部分人能做到严格控制饮食。而由于膳食营 养教育的缺失亦或是时间的限制, 许多子女无法帮助家中 的老人进行膳食搭配。

iii. 市场的呼声

在516名受访者中，有326名（63\%）受访者表示希望 有专业人员帮助订做家中老人的日常饮食。由此可以看出, 中国绝大部分家庭对专业膳食搭配有着一定的需求。专业 配餐是未来家庭的一大需求, 配餐市场潜力十分巨大。

您认为家里老人每天的饮食需不需要专业 人员给予量身定做?

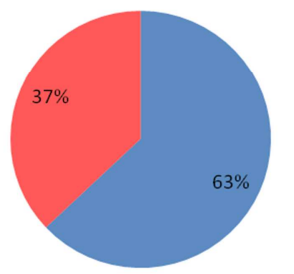

四需要 回不需要

图5 普通家庭专业饮食定做需求图。 
但事实上，受制于终端和社区医院的水平，中国的社 区医疗保障系统以及家庭配餐模式有以下缺点: 1. 老年人 对社区居家养老资源有效支付能力不足 2 . 社区居家人力 养老资源不足 3 . 社区养老服务资源整合不足 [7]

由于这些缺点, 家庭专业配餐和健康管理系统在中国 迟迟未能建立。但随着互联网的不断发展, 将互联网+跟 老年人健康管理相结合是实现社区居家养老, 管理老年人 健康的最佳途径。

\section{3. 系统开发方案}

\section{1 . 理论支持}

实施健康干预要遵循一定的步骤, 首先是建立一个研 究小组, 将选择的健康指导方针翻译成更明确的课程计划 和目标, 建立一个干预与自我效能感的理论框架。研究小 组包括营养师、行为心理学家, 一个公共卫生专家, 内分 泌专家和流行病学家。对这些健康准则确定干预的适宜含 量。然后根据相关的本地社区和老年人量身定制以适应当 地的情况下。一般来讲这包括了一套一般性的建议, 和至 少包含三个干预目标的课程计划。[8]这一过程应当包含: 1. 识别问题, 选择相对应的知识资料库 2 . 将具体知识运用 至具体问题上 3 . 评估知识的使用障碍 4 . 选择, 调整, 实施 干预措施 5. 监管知识运用 6 . 评估成果 7. 维持知识运用 [9]

综合这些干预与自我效能感的理论框架不仅在预测 和促进病人行为改变得到了验证, 也被证明可以实现临床 预后 [10]。例如, 在糖尿病患者的自我管理教育计划实验 中, 这一干预理论就被证明对提高患者的知识, 健康行为 以及自我效能有着明显的绩效。（自我效能指的是 “一个 人去组织和执行的自身行为的信念和相信一定会有成就 的决心”。它来自于一个人的成功或失败, 以及他人的经 验。）只有了在科学合理的知识体系支撑以及专业人员的 支援后, 互联网十健康才有了实体的意义, 才能被赋予它 应有的功能。

\section{2. 硬件支持}

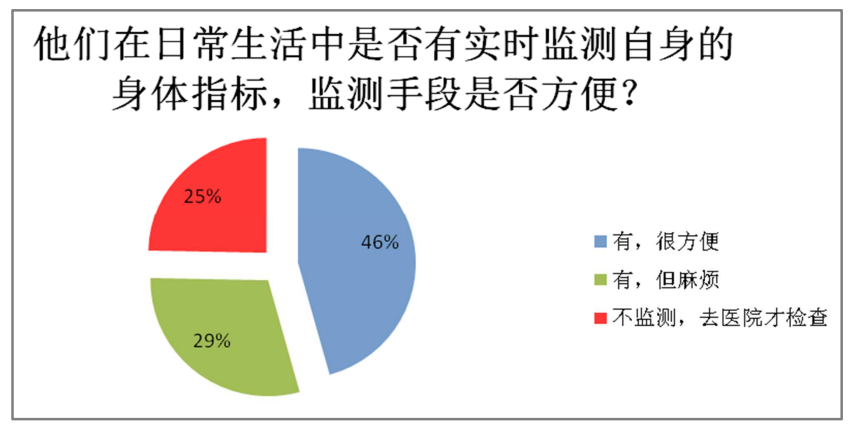

图6 老年人身体指标检测比例图。

市面上已经出现了许多方便快捷的监测身体指标的 终端, 如家用血糖仪, 血脂仪, 血压仪以及BMI体重秤等 等。但根据调查结果:

在516名调查者中仍然有 130 个家庭家中的老年人在 日常生活中没有实时监控身体指标, 同时还有149名调查
对象表示监控身体指标的终端不够方便。作为子女都难以 理解使用方法和数值, 更别说老年人了。老年人对于身体 的掌控情况不足很大程度上是因为终端不够友好, 难以操 作。所以在开发该系统时, 设备终端的轻便和易于使用应 当是产品开发的核心。而可穿戴设备的崛起为此提供了无 限可能。

可穿戴设备即指直接穿在身上, 或是整合到用户的衣 服或配件的一种便携式设备, 它不仅仅是一种硬件设备, 更是通过软件支持以及数据交互、云端交互来实现强大的 功能, 可穿戴设备将会对我们的生活、感知带来很大的转 变。[11]根据MaetzlerW [12] KrügerR在德国对帕金森患者 的研究 (帕金森病PD是一种常见的神经系统变性疾病, 老 年人多见, 平均发病年龄为 60 岁左右）, 可穿戴设备在医 疗行业中已经得到极佳的运用。由于其在健康和健身领域 的快速发展, 越来越多样的可穿戴设备在临床和家庭环境 中可以发挥着帕金森病症状的补体诊断作用。这种发展相 比于临床诊断具有明显的优势, 因为后者是相对主观的, 耗时和昂贵的, 并只提供了一个快照的条件。对PD症状的 评估使用这种技术的第一个结果 (包括运动障碍、震㗜、 运动迟缓、日常活动和睡眠行为）在家庭环境中是有极大 医疗价值的的。MaetzlerW认为, 这些技术可以提供PD患 者症状的补充信息, 并已被列入未来的诊断、检查、常规 治疗PD的技术列表中。这种技术的使用为病人更积极地参 与进医疗决策过程提供了机会。

通过可穿戴设备实时监测老年人的身体状况, 终端设 备可以监测以下常规数据: 步数, 步行距离, 用力呼吸量, 锻炼分钟数, 基础体温, 点滴出血, 宫颈粘液质量, 心率, 血压, 血糖, 血脂, 摔倒次数以及睡眠质量。老年人或者 子女可以通过端口 (App或者网页) 手动输入以下数据: 身高, 体重, 年龄, 三餐, 肺活量。

最后通过云端将所有数据进行整合, 在专业研究小组 的支持下提供合适的餐饮建议以及日常行为提示, 定期生 成健康报告, 在子女的手机或者网页上实时显示。让子女 轻松管理老年人健康, 实现互联网与健康管理的深度结合。

\section{3. 功能设计}

\subsection{1. 噮食搭配}

做一套健康管理系统，首先应当是膳食搭配的建议以 及营养餐的配送, 根据调查可知:

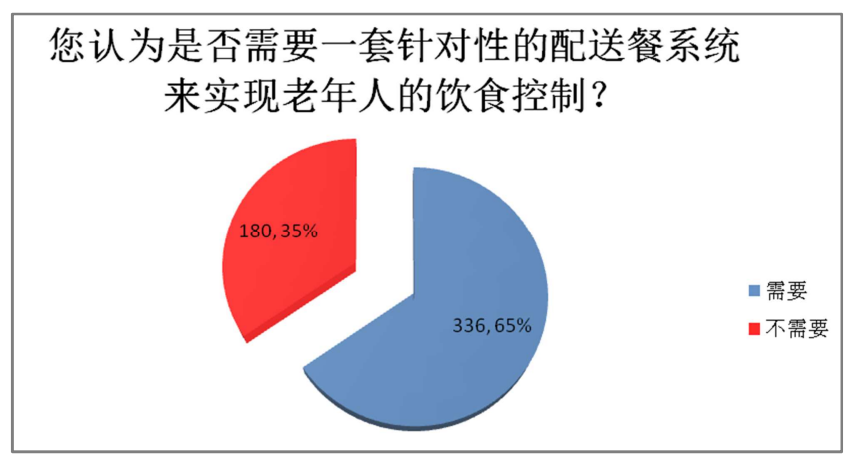

图7 配送餐系统市场需求图。 
有 336 个家庭 (65\%) 都认为老年人的饮食控制需要有 一套针对性的配送餐系统。因此为老年人配送日常三餐应 当是该系统的核心功能, 研究小组应当通过分析云端的数 据给每一个老年人制订相应的菜谱, 以在满足每个老人每 天的膳食营养需求的同时, 帮助老人减少高油高热量食品 的摄入, 只有通过严格管理好 “吃” ，才能真正管理好老 年人的身体健康。

\section{3. 2. 心理健康}

根据近年来学者的研究, 老年人的心理问题随着年 龄增长呈现多发或严重趋势, 这可能与随着年龄增长而 来的生理功能下降、患病率增高以及对死亡的恐惧有关 [13]。各研究领域学者针对性别与老年人心理健康的相 关性的研究结果是不一样的, 但冊庸置疑的是老年人的 心理健康会直接影响到老年人的身体健康。而老年人的 心理健康离不开自我管理、家庭支持和社会支持三个方 面, 从个人、家庭、社会多角度出发, 建立多元化、多 支柱的心理健康促进体系, 可以改善老年人心理健康 [14]。健康管理是通过对人们的身体和心理进行全方位 的检查、评价、剖析, 为其健康征询提出建议并对危害 健康的因素实施一系列干预的过程, 健康管理可以使人 们乃至全社会都积极行动, 充分利用现有资源使其发挥 最大化的作用 [15]。

根据调查结果可以看到: 有 $78 \%$ 的家庭认为受疾病困 扰的老年人在日常生活中需要心理指引和咨询。维持老年 人的心理健康是进行老年人健康管理的关键。

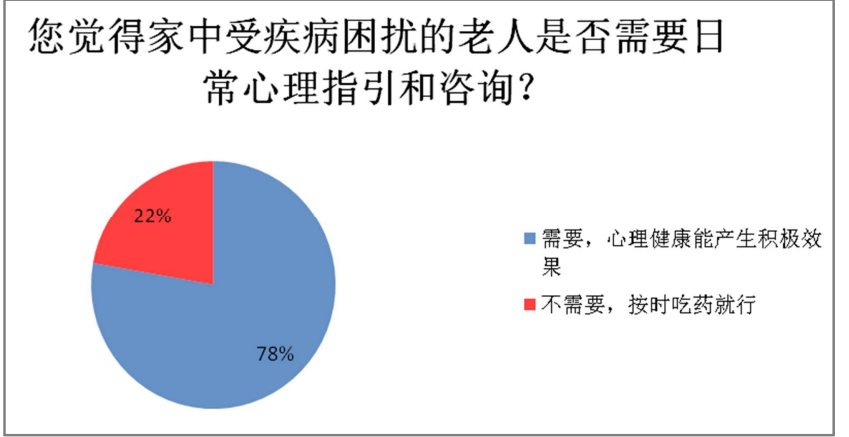

图8 老年人心理咨询需求比例图。

\section{3. 3. 社区合作}

社区是老年人购买养老服务产品最方便最快捷的场 所, 社区居家养老是以社区为依托, 由政府和社会机构 提供照顾日常生活、医疗保健、心理咨询、娱乐等方面 的服务, 老年人仍然居住在自己家中, 与亲人和朋友保 持原有的联系。[16]社区居家养老可以满足老年人的不 同需求, 为老年人节省住在养老机构的成本和费用, 能 够更好地利用我国现有的养老资源，以更灵活的时间和 方式提供养老服务。让该系统与社区相连接, 由线下商 家企业提供服务，受政府支持包括医疗保险的合约提供 以及社区药店合作提供上门服务等形式，全方位的实现 社区养老和互联网的结合。让老年人足不出户就享受到 专业医疗服务。

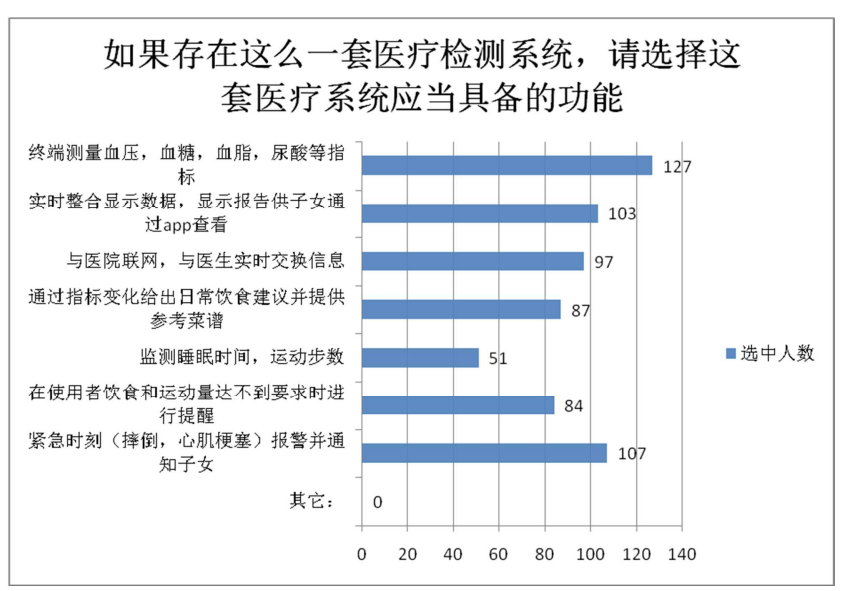

图9 医疗系统功能需求图。

根据调查结果可以发现, 这套系统应该包括终端测量 血压, 血糖, 血脂, 尿酸等指标, 实时整合显示数据, 显 示报告供子女通过app查看, 与医院联网, 与医生实时交 换信息, 通过指标变化给出日常饮食建议并提供参考菜谱, 监测睡眠时间, 运动步数, 在使用者饮食和运动量达不到 要求时进行提醒, 紧急时刻 (摔倒, 心肌梗塞) 报警并通 知子女等功能, 通过专业研究小组, 可穿戴式设备, APP, 数据整合实时监控老年人的健康并进行引导, 只有这样才 能真正把互联网与健康管理牢牢结合起来, 为老年人保驾 护航。

\section{3. 4. 系统定价}

一样产品是否具有实用性和推广性, 市场前景是否广 阔, 不仅仅是由它的功能决定的, 还要适当考虑价格。将 终端与健康数据相结合的系统, 虽然能让老年人和子女轻 松又有效的管理老年人的健康, 但如果价格让大部分家庭 无法承受的, 这套系统就失去其意义。

根据调查结果:

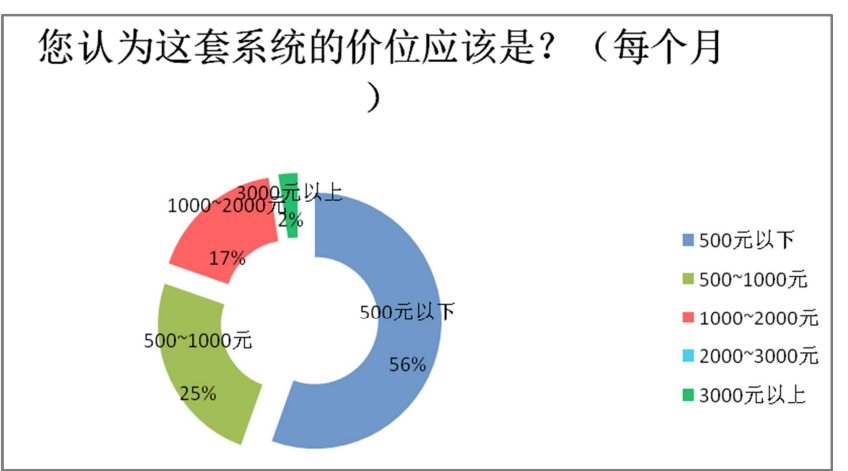

图10 医疗系统定价图。

有 $17 \%$ 的家庭愿意每个月出1000-2000元去使用该系 统, 而有 $25 \%$ 的家庭接受每个月500-1000元的健康管理开 销, 但超过半数的调查对象都希望系统价格在 500 元以下, 这也意味着在进行系统开发的时候需要尽可能压低成本, 以适应大部分家庭的消费水平, 从而真正让这个系统造福 老年人, 实现其社会意义。 


\section{4. 结论}

目前中国已全面步入老龄化社会, 老龄化将带给中国 经济和政策层面的严峻挑战, 老年人的健康管理关系着每 一个家庭。根据调查结果显示, 大部分家庭拥有 1 到 4 个老 人, 老年人数量以及所占的人口比例在逐年上升。慢性老 年疾病如高血糖, 高血压, 高血压, 心脏病等正困扰及影 响每个家庭的生活。有超过半数的家庭由于缺乏基本的医 疗饮食保障和知识, 正担心着老年人的日常饮食不当会对 老年人身体造成危害, 同时中国绝大部分家庭对专业膳食 搭配有着较大的需求。

本论文根据问卷调查结果, 结合目前互联网发展势头, 分析提出一个解决老年健康问题管理的可行性办法, 即设 计一套基于互联网的健康管理系统。互联网+健康管理是 符合社会需求的, 有很大潜力及发展空间。这套系统通过 专业研究小组, 可穿戴式设备, APP, 数据整合实时监控 老年人的健康并进行引导, 包括终端测量血压, 血糖, 血 脂, 尿酸等指标, 实时整合显示数据, 显示报告供子女通 过app查看, 与医院联网, 与医生实时交换信息, 通过指 标变化给出日常饮食建议并提供参考菜谱, 监测睡眠时间, 运动步数, 在使用者饮食和运动量达不到要求时进行提醒, 紧急时刻 (摔倒, 心肌梗塞) 报警并通知子女等功能。而 这套系统的价格应当被设计在每个月500元左右, 以适应 大部分家庭的消费水平。通过以上种种, 才能真正实现互 联网与健康管理的牢牢结合, 为老年人健康保驾护航。

\section{致谢}

本文为2016江苏省大学生创新创业训练计划省级指 导项目《将互联网终端引入老年人健康管理的方法研究》 的阶段性成果之一。

\section{参考文献}

[1] 中国统计局中国统计年鉴2015年

２］张璐, 孔灵芝. 预防慢性病: 一项至关重要的投资一一世界 卫生组织报告明 [J]. 中国慢性病预防与控 制, 2006, 14 (1) : 1
[3] 刘盼盼, 王红妹, 郭岸英. 以社区为基础的常见慢性病疾病 管理 [J]. 中华医院管理杂志. 2010, 26 (3) : 207-209

[4] 深圳中医_深圳固生堂官网三高之间的互相影响 2013-11-18

[5] 搜狐科技5分钟带你了解中国互联网现状！2015-12-14

[6] 丁香园基层医疗和「互联网+」能否成为慢病管理的突破口? 2016-05-31

［7］常媛媛人口老龄化背景下构建社区居家养老模式的路径分 析2016-3

[8] Amutha Ramadas \& Carina Ka Yee ChanAWeb-Based Dietary Intervention for People with Type 2Diabetes: Development, Implementation, and Evaluation DOI 10. 1007/s12529-014-9445-z

[9] Catherine H Yu, Janet Parsons, Muhammad Mamdani Designing and evaluating a web-based selfmanagement site for patients with type 2diabetes - systematic website development and study protocol Yu et al. BMC Medical Informatics and Decision Making 2012, 12:57

[10] Nakahara R, Yoshiuchi K, Kumano H, Hara Y, Suematsu $\mathrm{H}$, Kuboki T: Prospective study on influence of psychosocial factors on glycemiccontrol in Japanese patients with type 2 diabetes. Psychosomatics 2006, $47: 240-5$

[11] 东莞日报 2015 中国可穿戴设备市场将达 114.9 亿 2016-01-15

[12] Maetzler W1, Krüger R1 Wearable Technique for the Assessment of Parkinson Symptoms: What's the Future? 2016 Jun 8

[13] 滕海英, 王倩云, 熊林平, 等. 西安市社区老年人心理健康状 况及其影响因素分析 $[\mathrm{N}]$. 第二军医大学学 报, 2012, 33(10) : 1095-1098

[14] 王大华, 张明妍. 老年人配偶支持的特点及其与夫妻依恋、 婚姻满意度的关系 [J]. 心理发展与教育, 2011, 2: 195-201

[15] 傅华, 王家骥, 李枫, 等. 健康管理的理论与实践 $[\mathrm{J}]$. 健康教 育及健康促进, 2007, 2 (3) : 16

[16] 张斯日古楞, 白明艳. 我国城镇社区养老服务存在的问题及 对策 $[\mathrm{J}]$. 劳动保障世界, 2014（1）：6-7 